\title{
Influence of laser pulse shaping on the ultrafast dynamics in antiferromagnetic $\mathrm{NiO}$
}

\author{
Andrea Rubano, ${ }^{1}$ Takuya Satoh, ${ }^{2,3}$ Alexey Kimel, ${ }^{4}$ Andrey Kirilyuk, ${ }^{4}$ Theo Rasing, ${ }^{4}$ and Manfred Fiebig ${ }^{1}$ \\ ${ }^{1}$ HISKP, Universität Bonn, Nussallee 14-16, 53115 Bonn, Germany \\ ${ }^{2}$ Institute of Industrial Science, The University of Tokyo, Komaba, Meguro-ku, Tokyo 153-8505, Japan \\ ${ }^{3}$ PRESTO, Japan Science and Technology Agency, Kawaguchi, Saitama 332-0012, Japan \\ ${ }^{4}$ Radboud University Nijmegen, Institute for Molecules and Materials, 6525 AJ Nijmegen, The Netherlands
}

(Received 13 July 2010; published 23 November 2010)

\begin{abstract}
Femtosecond laser excitation of antiferromagnetic NiO leads to an oscillatory temporal behavior of optical second-harmonic generation in this material. Depending on laser pulse length, these oscillations have frequencies of either 55 or $1 \mathrm{GHz}$, which correspond to the magnetic anisotropy energy separating the ground state with spins along the $\langle 11 \overline{2}\rangle$ axis from the excited states with spins along the $\langle 111\rangle$ or $\langle 1 \overline{1} 0\rangle$ axis, respectively. The oscillations can be explained as beatings between the ground and the respective excited state triggered by an instantaneous laser-induced flip of the $\mathrm{Ni}^{2+}$ spins which can thus be controlled three dimensionally by pulse shaping.
\end{abstract}

DOI: 10.1103/PhysRevB.82.174431

PACS number(s): 78.47.- p, 42.65.Ky, 75.30.Gw, 75.50.Ee

\section{INTRODUCTION}

One of the emerging approaches of magnetic memory applications is ultrafast optical manipulation of spins in magnetically ordered materials. ${ }^{1}$ Over the last 20 years considerable progress has been achieved in understanding the fundamental time scales for the speed of magnetization reversal in ferromagnet and ferrimagnet. ${ }^{2}$ Although magnetization reversal in these materials at a subpicosecond time scale seems to be feasible, ${ }^{3,4}$ it has also been realized that spin reorientation in antiferromagnets may occur at a much faster time scale than that in ferromagnets, ${ }^{5-7}$ because of the direct involvement of the exchange interaction between the sublattices making up the antiferromagnetic (AFM) state.

Experimental observation of spin reorientation over $90^{\circ}$ in an antiferromagnet has been claimed in only three compounds thus far: $\mathrm{NiO}_{2}{ }^{8} \mathrm{TmFeO}_{3},{ }^{9}$ and $\mathrm{HoFeO}_{3} \cdot{ }^{10} \mathrm{In} \mathrm{TmFeO}_{3}$ and $\mathrm{HoFeO}_{3}$ the reorientation occurs between two equilibrium states of the materials. The transition is observed via linear magneto-optical effects that couple directly to the AFM order parameter. The spin reorientation is followed by spin precession with the frequency of the magnetic resonance. The manifestation of the photoinduced spin reorientation in $\mathrm{NiO}$ is quite different. After the photoexcitation by a $\sim 100$ fs laser pulse, the second-harmonic generation (SHG) probe signal showed a $55 \mathrm{GHz}$ oscillation that, however, did not match any of the known collective spin excitations of the material and was explained as interference effect.

In this paper we aim to advance our understanding of the ultrafast SHG response of photoexcited NiO. We show that the temporal behavior of $\mathrm{SHG}$ from photoexcited $\mathrm{NiO}$ can be controlled by varying the pulse length of the excitation laser. In particular, a fine tuning of the pulse width leads to an abrupt and dramatic change in the oscillation frequency. These observations can be explained by assuming that the laser pulse causes an instantaneous flip of the $\mathrm{Ni}^{2+}$ spins from the $\langle 11 \overline{2}\rangle$ ground state into the $\langle 111\rangle$ hard axis or into the $\langle 1 \overline{1} 0\rangle$ direction within the easy plane. Due to beatings, the SHG signal shows oscillations with the frequencies determined by the energy separation of either the $\langle 11 \overline{2}\rangle$ and $\langle 111\rangle$ states $(55 \mathrm{GHz})$ or the $\langle 11 \overline{2}\rangle$ and $\langle 1 \overline{1} 0\rangle$ states $(1 \mathrm{GHz})$. The measurements in the time domain also provide an approach for probing the corresponding magnetic anisotropy energies with unprecedented resolution.

\section{PROBING AFM NiO BY SHG}

$\mathrm{NiO}$ is an insulating material with a compensated AFM structure. Because of its high Néel temperature of $T_{N}$ $=523 \mathrm{~K},{ }^{11}$ it is a promising exchange-bias material. In the paramagnetic state, $\mathrm{NiO}$ has a $\mathrm{NaCl}$-like cubic structure with $m 3 m$ point symmetry. At $523 \mathrm{~K}_{\text {the }} \mathrm{Ni}^{2+}$ spins become confined to $\{111\}$ planes, where they are ordered ferromagnetically along $\langle 11 \overline{2}\rangle$ axes. Opposite spin orientation in neighboring $\{111\}$ planes makes the compound AFM. NiO is an easy-plane system. The hard axis is $\langle 111\rangle$, i.e., the stacking direction of the ferromagnetic planes. It is separated by an anisotropy energy $\hbar D_{\text {hard }}=100 \pm 10 \mu \mathrm{eV}$ from the easy plane in which the much weaker anisotropy energy $\hbar D_{\text {easy }}$ $=5 \pm 4 \mu \mathrm{eV}$ distinguishes the $\langle 11 \overline{2}\rangle$ and the $\langle 1 \overline{1} 0\rangle$ axes and determines the spin direction. ${ }^{12-14}$

SHG has been demonstrated to be an effective tool for probing the magnetic state in ferromagnetic ${ }^{15}$ and, in particular, in AFM materials. ${ }^{16}$ SHG is a second-order optical process in which an incident light field at frequency $\omega$ interacts with the material to generate a polarization at frequency $2 \omega$. The coupling term is the second-order susceptibility tensor $\hat{\chi}^{(2)}$ which, in the case of $\mathrm{NiO}$, depends on the square of the AFM order parameter. ${ }^{17}$ This coupling was employed to investigate the electronic transitions, the AFM domain structure, and the magnetization dynamics of this compound. ${ }^{8,17}$

In the present experiment, polished (111)-cut $\mathrm{NiO}$ samples were prepared from single crystals grown by the flame-fusion method and annealed in oxygen in order to obtain a domain size of $\sim 100 \mu \mathrm{m}$. Pump pulses of about 100 fs were generated by an amplified Ti:sapphire laser with a photon energy of $1.55 \mathrm{eV}$. Probe pulses were generated by an optical parametric amplifier at a photon energy of $1.03 \mathrm{eV}$ where the SHG yield reaches a spectral maximum. ${ }^{17}$ The 


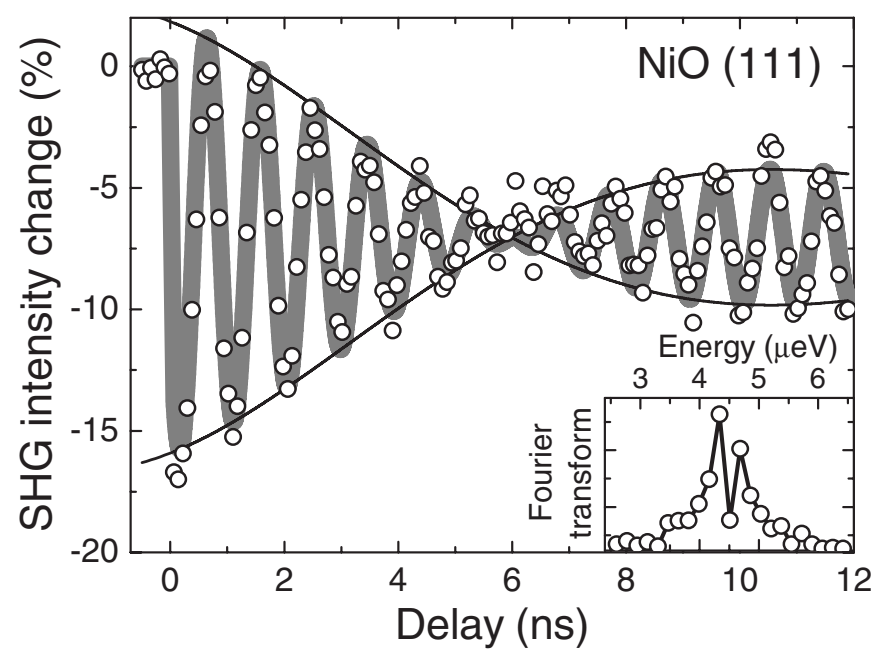

FIG. 1. Temporal evolution of the SHG intensity of a NiO (111) single crystal induced by optical pumping. The change in SHG intensity is shown in dependence of the delay to the photoexcitation at $t=0$. Data were taken at room temperature. The gray thick line and the envelope (black lines) were derived by fitting Eq. (1). The inset shows the Fourier-transformed SHG data, after deconvolution of the exponential decay.

probe pulse was sent through a mechanical delay line for generating a retardation of up to $12 \mathrm{~ns}$. It was focused onto a single domain of the sample with a focus diameter of about $50 \mu \mathrm{m}$. Pump and probe pulses differed in energy by a factor 20 and in diameter by a factor 3. SHG was generated in near-normal-incidence reflection and detected as described elsewhere. ${ }^{8,17}$

\section{PUMP-AND-PROBE SHG EXPERIMENTS ON NiO}

\section{A. Probe-signal beating}

In Fig. 1 the relative change in the SHG yield upon exciting the $\mathrm{NiO}$ sample with the pump beam is shown as a function of the delay between the arrival of the pump and the probe pulse. Two features characterize the evolution of the SHG signal at $t>0$. First, a drop of the average SHG intensity by about $7 \%$ occurs within the duration of the pump pulse. Second, the SHG signal exhibits a pronounced oscillation with a frequency of about $1 \mathrm{GHz}$. The amplitude of the oscillation is modulated by beating and an exponential decay. It was verified that neither the drop nor the modulated oscillation of the SHG signal is reproduced by the simultaneously measured reflectivity at frequency $\omega$.

The evolution of the SHG intensity change $\Delta I(t)$ at $t>0$ can be described as follows:

$$
\Delta I(t)=\Delta I_{0}+A \exp (-t / \tau) \cos (\Omega t) \sin (\Delta \Omega t) .
$$

Here, $\tau$ represents the decay time of the SHG oscillation while $\Omega$ and $\Delta \Omega$ denote its mean frequency and the doublet splitting, respectively, according to the Fourier transformation in the inset of Fig. 1. A fit reveals $\tau=9.7 \pm 0.2 \mathrm{~ns}, \hbar \Omega$ $=4.39 \pm 0.08 \mu \mathrm{eV}$, and $\hbar \Delta \Omega=0.172 \pm 0.002 \mu \mathrm{eV}$, a value that lies almost seven orders of magnitude below the excitation photon energy used in this experiment. Surprisingly, the oscillation frequency of the SHG intensity triggered by the photoexcitation does not match any known collective excitation of spins or lattice in $\mathrm{NiO}$. It is also clear that optical decoherence processes are operating at a subpicosecond time scale and cannot explain oscillations of an optical signal in the nanosecond regime.

A similar situation was reported in Ref. 8. There it was proposed that optical pumping leads to a transient change in the magnetic anisotropy of the $\mathrm{Ni}^{2+}\left(3 d^{8}\right)$ ion so that the $\mathrm{Ni}^{2+}$ spins flip collectively and instantaneously from the $\langle 11 \overline{2}\rangle$ to the $\langle 111\rangle$ direction while maintaining their AFM order. This situation is illustrated by the light gray curve in Fig. 3(a). It visualizes a situation where pronounced photoexcitation initiates a transient change in the magnetic easy direction from $\langle 11 \overline{2}\rangle$ to $\langle 111\rangle$ so that a proposed spin flip can occur. As further detailed in Ref. 8, subsequent SHG on the $\langle 11 \overline{2}\rangle$ and $\langle 111\rangle$ states lead to three SHG contributions at $2 \omega$ and $2 \omega \pm D_{\text {hard }}$. Interference of these contributions leads to beating in the intensity of the SHG signal at $2 \hbar D_{\text {hard }}$ (with a small admixture at $\hbar D_{\text {hard }}$ ).

The SHG oscillation in Fig. 1 is reminiscent of this behavior but distinct discrepancies to the earlier result are obvious: (i) the additional modulation of the SHG oscillation and the exponential decay were not found in the earlier work. (ii) The period $\Omega^{-1}$ of the oscillation is more than an order of magnitude longer than the one obtained in Ref. 8, whereas the former SHG oscillation is not observed. Yet, the value $\hbar \Omega=4.39 \pm 0.08 \mu \mathrm{eV}$ is of the same order as $2 \hbar D_{\text {easy }}$ with $\hbar D_{\text {easy }}=5 \pm 4 \mu \mathrm{eV}$ as anisotropy energy separating the spin directions $\langle 11 \overline{2}\rangle$ and $\langle 1 \overline{1} 0\rangle$ according to inelastic neutron scattering. ${ }^{12}$ The coincidence between $\Omega / 2$ and $D_{\text {easy }}$ becomes even more striking in the view of recent magnon excitation experiments. ${ }^{18}$ They reveal a ratio of magnetic in-plane and out-of-plane anisotropies of $D_{\text {easy }} / D_{\text {hard }}=0.017 \pm 0.002$ while we obtain $(\Omega / 2) / D_{\text {hard }}$ $=0.019 \pm 0.002$ in the present experiment. The question is why, under similar conditions as in Ref. 8, such striking differences are obtained in the ultrafast response of photoexcited $\mathrm{NiO}$ in the present experiment and how they can be explained.

\section{B. Pump-pulse tuning}

Figure 2 shows how the temporal evolution of the photoinduced change in the SHG intensity depends on the duration of the pump pulse. A pulse length of $130 \mathrm{fs}$ (top) and $110 \mathrm{fs}$ (bottom) was set by slightly tuning the pulse compression. However, for all plots shown in Fig. 2 the energy of the pump pulse and, hence, the laser fluence on the sample, were kept constant in order to prevent any effects related to the laser pulse energy. Figure 2 reveals that the relatively small change in the pump-pulse parameters has a dramatic effect on the observed dynamics. With the $130 \mathrm{fs}$ pulse the same long-term oscillation as in Fig. 1 is obtained, and the shortterm oscillation of Ref. 8 is absent. In contrast, the $110 \mathrm{fs}$ pulse reveals the short-term oscillation while the long-term oscillation is not present anymore.

First of all, this behavior is consistent with the model of a photoinduced instantaneous reorientation of the AFM spins. 


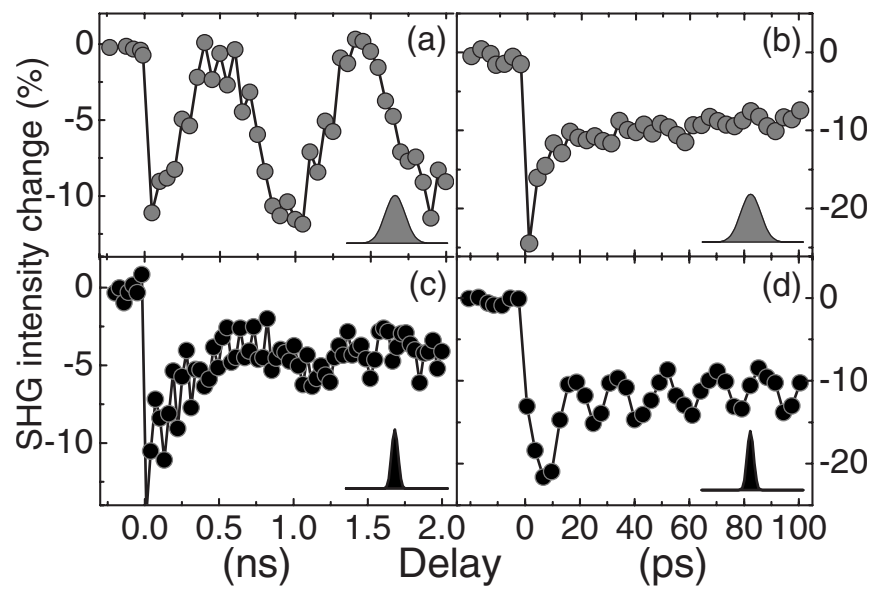

FIG. 2. Temporal evolution of the SHG signal at fixed laser fluence and different pump pulse durations $\tau_{P}$. [(a) and (b)] $\tau_{P}=130 \mathrm{fs}$; [(c) and (d)] $\tau_{P}=110$ fs. Data were taken at $80 \mathrm{~K}$.

Such an excitation can flip the spins into either direction: within or out of the easy plane, but not both. This is illustrated by Fig. 3(a). Depending on the strength of the photoexcitation, either the $\langle 1 \overline{1} 0\rangle$ (dark gray curve) or the $\langle 111\rangle$ (light gray curve) direction can become the transient easy axis. Consequently, only one of the two SHG oscillations can be present at a time. Second, since the experiment was done at a constant laser fluence and the pulse lengths were far below the spin-lattice relaxation time, the data shown in Fig. 2 cannot be understood in terms of laser-induced heating of $\mathrm{NiO}$. The mechanism responsible for the excitation of the oscillations therefore must have an entirely nonthermal origin.

For understanding the behavior illustrated by Fig. 2, we have to recall that the observed phenomenon is a threshold process. ${ }^{8}$ As Fig. 3(a) shows, the photoexcitation has to surpass the point where the magnetic anisotropy acquires a new, transient, easy direction as a prerequisite for the flip of the $\mathrm{Ni}^{2+}$ spins $^{8}$ (dark or light gray curve). Exceeding this threshold is a matter of balance between the number of electrons displaced by the pump pulse and the number of carriers returning to the ground state via electron relaxation.

This is described by the simulation shown in Fig. 3(b). The simulation is based on a rate equation for the density of photoexcited electrons $n(t)$,

$$
\frac{d n}{d t}=\alpha G(t)-\frac{n}{\tau_{e}},
$$

where $\alpha$ is the optical absorption and $\tau_{e}$ the electron relaxation time. $G(t)=\left(G_{0} / \sqrt{2 \pi \sigma^{2}}\right) \exp \left[-(t / \sigma)^{2}\right]$ describes the Gaussian pump pulse with $\sigma$ as pulse length and $G_{0}$ proportional to the pulse energy. This differential equation is solved by

$$
n(t)=\frac{\alpha}{2} G_{0} e^{(\sigma / 2 \tau)^{2}}\left(\left[\operatorname{erf}\left(\frac{t}{\sigma}-\frac{\sigma}{2 \tau}\right)+1\right]\right) e^{-t / \tau},
$$

where $\operatorname{erf}(x)=(2 / \sqrt{\pi}) \int_{0}^{x} \exp \left(-y^{2}\right) d y$ is the Gaussian error function. In Fig. 3(b), the behavior of $n(t)$ for two extreme
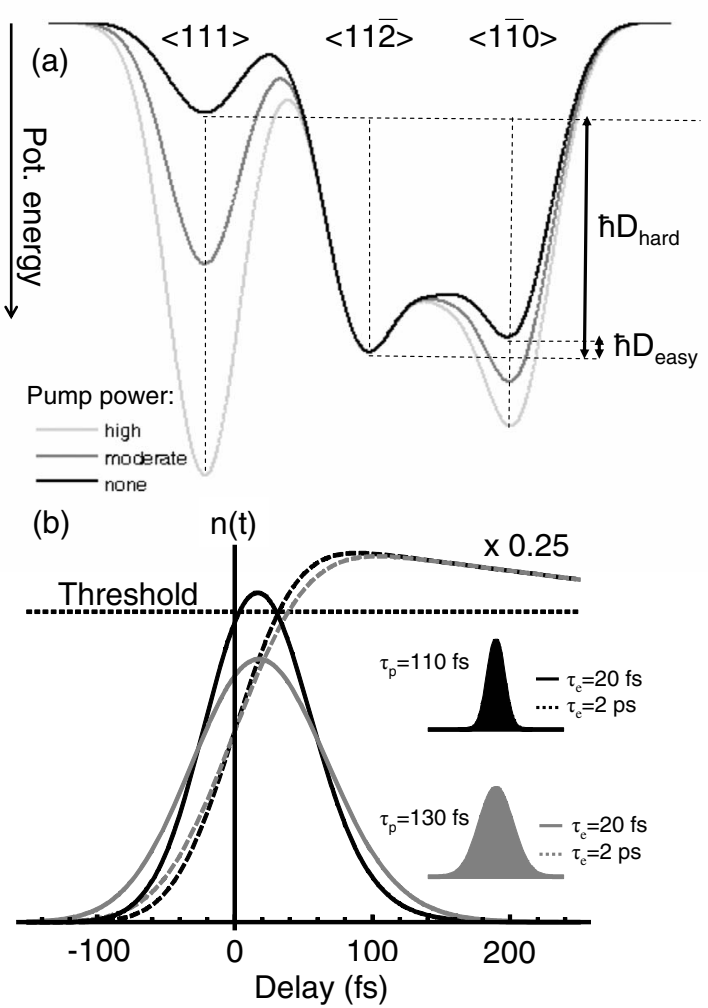

FIG. 3. Model for the photoinduced processes observed in NiO. (a) Sketch of the proposed change in magnetic anisotropy from its ground state (black curve) by the presence of the optical pump pulse. The energy of the $\langle 11 \overline{2}\rangle$ state was taken as reference value. Once the excitation becomes sufficiently strong, $\langle 1 \overline{1} 0\rangle$ (dark gray curve) or $\langle 111\rangle$ (light gray curve) may become the transient new easy direction for the $\mathrm{Ni}^{2+}$ spins and a collective spin flip occurs. (b) Simulation based on Eq. (3) highlighting the influence of the electron-electron relaxation time $\tau_{e}$ and the pump pulse duration $\tau_{P}$ on the photoexcited density of carriers $n(t)$. Solid and dashed lines refer to $\tau_{e}=20$ fs and $\tau_{e}=2 \mathrm{ps}$, respectively; black and gray lines refer to $\tau_{P}=110$ fs and $\tau_{P}=130 \mathrm{fs}$, respectively. The threshold was arbitrarily entered in order to illustrate the experimental observation: with $\tau_{P}=110$ fs the density for a spin flip into the $\langle 111\rangle$ direction is reached while it is missed for $\tau_{P}=130 \mathrm{fs}$.

cases is shown in order to illustrate the role of the electron relaxation time in our experiment. We assume an electron relaxation time that is much shorter (20 fs, solid lines) or much longer ( $2 \mathrm{ps}$, dashed lines) than the laser pulse length. In the fast-decay scenario, the pulse duration plays a critical role in overcoming the threshold for the hard-axis reorientation while in the slow-decay scenario, the influence of the pulse duration is negligible. Comparison of Fig. 3(b) with Fig. 2 clearly points to the former scenario, consistent with an electron relaxation time $\ll 100$ fs for $\mathrm{NiO}$, as was suggested in Ref. 8. With respect to Fig. 2, we can therefore conclude that the energy we provide to the system using the 110 fs pump pulse is sufficient for overcoming the threshold for spin reorientation out of the easy plane and along the $\langle 111\rangle$ axis and, thus, the corresponding beating is seen in Fig. 2(d) and gives $\hbar D_{\text {hard }}=114.8 \pm 0.3 \mu \mathrm{eV}$ as value of the anisotropy energy. By increasing the pulse duration to $130 \mathrm{fs}$, 
the electronic relaxation gains weight and even though the pulse energy is not changed the threshold for a spin flip out of the easy plane is no longer reached. Instead, SHG beating as in Fig. 2(a) is obtained, which points to a spin flip within the easy plane from the $\langle 11 \overline{2}\rangle$ to the $\langle 1 \overline{1} 0\rangle$ axis. With $D_{\text {hard }} / D_{\text {easy }} \simeq 50$, the threshold for the latter process will be relatively low. Figure 2(a) gives $\hbar D_{\text {easy }}=2.20 \pm 0.04 \mu \mathrm{eV}$ as corresponding value of the anisotropy energy in agreement but with a much smaller error than in Ref. 12.

Note that the change in frequency of the SHG oscillation in Fig. 2 can also be triggered by tuning the laser fluence while keeping the width of the laser pulse unchanged. However, this would disguise the independence of heating effects that we want to emphasize. In addition, the SHG oscillation is more sensitive to tuning the pulse width than it is to a change in fluence which indicates that pump-dump effects in the stretched laser pulse ${ }^{19}$ may also affect the density of photoexcited electrons $n(t)$.

\section{DISCUSSION OF MICROSCOPIC MECHANISMS}

The oscillatory SHG response of $\mathrm{NiO}$ subsequent to photoexcitation is thus consistent with an ultrafast reorientation of the AFM spins, where the details of the reorientation strongly depend on the length of the pump pulse according to Fig. 3. A microscopic justification of this effect must relate the optical excitation of the $\mathrm{Ni}^{2+}\left(3 d^{8}\right)$ electrons to a modification of the ground state of the electronic system by a change in the magnetic anisotropy as sketched in Fig. 3(a). The optical excitation shifts electrons between the $3 d$ states of the $\mathrm{Ni}^{2+}$ that are split by the ligand field, spin-orbit interaction, and exchange effects. ${ }^{17}$ We have to assume that: (i) the repopulation modifies the anisotropy to such an extent that a transient change in the easy axis occurs which provokes the $90^{\circ}$ spin flip; (ii) to see the beatings, the spin reorientation itself must occur within the duration of the pump-laser pulse. Assumption (i) can be justified. Though the exact mechanism of this modification with the electronic processes on the atomic level of the $\mathrm{Ni}^{2+}$ ion is not yet understood it is known that the easy axis of $\mathrm{NiO}$ can be changed relatively easily by a magnetic field, ${ }^{20}$ by $\mathrm{Co}$ deposition, ${ }^{21}$ or by pressure. ${ }^{22}$ In addition the holes generated by the photoinduced excitation of electrons may lead to the formation of Zheng-Rice-type singlet states that were shown to modify the spin direction while retaining the antiferromagnetic order. ${ }^{23}$ Regarding assumption (ii), reorientation of an AFM order parameter in the presence of photoexcitation was discussed in Refs. 5-7. Note that we do not pump the
$\mathrm{NiO}$ resonantly and an influence of the pump polarization is not observed so that a different scenario than in the former theoretical work has to be considered in our case.

The pronounced and long lasting beating effects observed in the experiment indicate that the interactions destroying this coherence are weak. The impressively long coherence time, following from Fig. 1, shows that the oscillations cannot be due to the optical coherence between the pump and probe light fields. ${ }^{24}$ In contrast, the spin system with its weak coupling to the lattice can remain sufficiently unperturbed for maintaining the long coherence times obtained here. Extending this rationale, we may conclude that the beating in Fig. 1 with the splitting revealed in the inset must also be spin related. Possible mechanisms falling in the same energy range as $\hbar \Delta \Omega$ are, for instance, hyperfine interactions, domain-wall motion, or the Zeeman splitting caused by perturbations in the order of the terrestrial magnetic field. The fact that we observe such beatings at room temperature corroborates a collective behavior of large number of spins. Otherwise temperature fluctuations $(\sim 25 \mathrm{meV})$ would easily destroy the coherence. However, more detailed theory is required to substantiate these suggestions, as well as to find the explicit microscopic mechanism of the instantaneous spin flip induced by the femtosecond pump pulse.

\section{CONCLUSION}

In summary, we have observed an oscillatory optical second-harmonic generation from $\mathrm{NiO}$ excited by femtosecond laser pulses. Depending on the laser pulse at fixed laser fluence oscillation frequencies of 55 or $1 \mathrm{GHz}$ are observed that are related to the out-of-plane or the in-plane anisotropy energy for a $\langle 11 \overline{2}\rangle \rightarrow\langle 111\rangle$ or $\langle 11 \overline{2}\rangle \rightarrow\langle 1 \overline{1} 0\rangle$ flip, respectively, of the $\mathrm{Ni}^{2+}$ spins. These results point to a nonthermal three-dimensional reorientation of the AFM spins of $\mathrm{NiO}$ by optical pumping with tailored femtosecond laser pulses. They also provide a rather sensitive method to probe these very small differences in the anisotropy energy revealing $\hbar D_{\text {hard }}=114.8 \pm 0.3 \mu \mathrm{eV}$ and $\hbar D_{\text {easy }}=2.20 \pm 0.04 \mu \mathrm{eV}$ in the present case.

\section{ACKNOWLEDGMENTS}

The research leading to these results has received funding from de Nederlandse Organizatie voor Wetenschappelijk Onderzoek (NWO), de Stichting voor Fundamenteel Onderzoek der Materie (FOM), and the Deutsche Forschungsgemeinschaft (DFG, through the priority program SPP1133). T.S. was supported by the Foundation for the Promotion of Industrial Science.
${ }^{1}$ B. Koopmans, in Spin Dynamics in Confined Magnetic Structures II, Topics in Applied Physics Vol. 87, edited by B. Hillebrands and K. Ounadjela (Springer, Berlin, 2003), pp. 256-323.

${ }^{2}$ J. Stöhr and H. C. Siegmann, Magnetism: From Fundamentals to Nanoscale Dynamics (Springer-Verlag, Berlin, 2006).

${ }^{3}$ S. J. Gamble, M. H. Burkhardt, A. Kashuba, R. Allenspach, S. S. P. Parkin, H. C. Siegmann, and J. Stöhr, Phys. Rev. Lett. 102,
217201 (2009)

${ }^{4}$ C. D. Stanciu, A. Tsukamoto, A. V. Kimel, F. Hansteen, A. Kirilyuk, A. Itoh, and T. Rasing, Phys. Rev. Lett. 99, 217204 (2007).

${ }^{5}$ R. Gómez-Abal, O. Ney, K. Satitkovitchai, and W. Hübner, Phys. Rev. Lett. 92, 227402 (2004).

${ }^{6}$ G. Lefkidis and W. Hübner, Phys. Rev. B 76, 014418 (2007). 
${ }^{7}$ G. Lefkidis, G. P. Zhang, and W. Hübner, Phys. Rev. Lett. 103, 217401 (2009).

${ }^{8}$ N. P. Duong, T. Satoh, and M. Fiebig, Phys. Rev. Lett. 93, 117402 (2004).

${ }^{9}$ A. V. Kimel, A. Kirilyuk, A. Tsvetkov, R. V. Pisarev, and Th. Rasing, Nature (London) 429, 850 (2004).

${ }^{10}$ A. V. Kimel, B. A. Ivanov, R. V. Pisarev, P. A. Usachev, A. Kirilyuk, and Th. Rasing, Nat. Phys. 5, 727 (2009).

${ }^{11}$ J. T. Richardson and W. O. Milligan, Phys. Rev. 102, 1289 (1956).

${ }^{12}$ M. T. Hutchings and E. J. Samuelsen, Phys. Rev. B 6, 3447 (1972).

${ }^{13}$ H. Kondoh, J. Phys. Soc. Jpn. 15, 1970 (1960).

${ }^{14}$ A. J. Sievers and M. Tinkham, Phys. Rev. 129, 1566 (1963).

${ }^{15}$ A. Kirilyuk and Th. Rasing, J. Opt. Soc. Am. B 22, 148 (2005).

${ }^{16}$ M. Fiebig, V. V. Pavlov, and R. V. Pisarev, J. Opt. Soc. Am. B 22, 96 (2005).

${ }^{17}$ M. Fiebig, D. Fröhlich, T. Lottermoser, V. V. Pavlov, R. V. Pisarev, and H. J. Weber, Phys. Rev. Lett. 87, 137202 (2001).
${ }^{18}$ T. Satoh, S.-J. Cho, R. Iida, T. Shimura, K. Kuroda, H. Ueda, Y. Ueda, B. A. Ivanov, F. Nori, and M. Fiebig, Phys. Rev. Lett. 105, 077402 (2010).

${ }^{19}$ P. Nuernberger, G. Vogt, T. Brixner, and G. Gerber, Phys. Chem. Chem. Phys. 9, 2470 (2007)

${ }^{20}$ S. Saito, M. Miura, and K. Kurosawa, J. Phys. C 13, 1513 (1980).

${ }^{21}$ H. Ohldag, A. Scholl, F. Nolting, S. Anders, F. U. Hillebrecht, and J. Stööhr, Phys. Rev. Lett. 86, 2878 (2001).

${ }^{22}$ H. Kondoh and T. Takeda, J. Phys. Soc. Jpn. 19, 2041 (1964).

${ }^{23}$ T. Haupricht, J. Weinen, S. G. Altendorf, Y.-Y. Chin, Z. Hu, J. Gegner, H. Fujiwara, D. Regesch, R. Gierth, A. Hendricks, H. H. Hsieh, H.-J. Lin, C. T. Chen, and L. H. Tjeng, International Conference on Magnetism, Karlsruhe, Germany, 2009 (unpublished).

${ }^{24}$ J. H. Versluis, A. V. Kimel, V. N. Gridnev, D. R. Yakovlev, G. Karczewski, T. Wojtowicz, J. Kossut, A. Kirilyuk, and Th. Rasing, J. Phys.: Condens. Matter 22, 115801 (2010). 